\title{
Augmenting SCORM Manifests with Adaptive Links
}

\author{
Nor Aniza Abdullah, Christopher Bailey and Hugh Davis \\ LTG \& IAM Research Groups, ECS \\ The University of Southampton \\ Southampton, UK \\ \{naa02r, cpb, hcd\}@ecs.soton.ac.uk
}

\begin{abstract}
This paper describes an experiment to augment SCORM manifests with adaptive links using AuldLinky in order to promote content reusability, interoperability and personalized elearning. Our technique involves the automatic deduction of a concept map from a manifest and the transformation of its pertinent elements into FOHM (Fundamental Object Hypermedia Model) objects before augmenting the information with complimentary and adaptive links using AuldLinky.
\end{abstract}

\section{Categories and Subject Descriptors}

H.5.4 [Information Interfaces and Presentation]: Hypertext/Hypermedia - Architecture, Navigation.

\section{General Terms}

Design, Human Factors, Standardization

\section{Keywords}

SCORM, Adaptive Hypermedia, AuldLinky, FOHM

\section{INTRODUCTION}

A common issue in E-Learning has been to provide mechanisms to allow the re-use and interoperability of learning resources. Recent advances in the area of Learning Objects have led to the adoption of SCORM, which consists of a manifest describing both the resources to be used for a particular unit of learning material (html, Flash, PDFs etc.) along with one or more organisations the order in which these resources will be presented or sequenced. SCORM courseware (the manifest and the resources) may then be packaged in a PIF (Package Interchange File) [1] in order to facilitate interchange between Learning Management Systems (LMSs).

A frequent criticism of the SCORM approach to courseware is that the pedagogy is simplistic and more suitable for training than for education. The most recent version of SCORM includes IMS Simple Sequencing [2] which allows the author to include assessment points and use the results to dictate the conditions under which a learner might progress to further material or be routed to remedial material. However this sequencing falls short of the aims of the adaptive hypermedia community to provide personalised e-Learning [3]; the user model is simplistic and does not persist from unit to unit.

Permission to make digital or hard copies of all or part of this work for personal or classroom use is granted without fee provided that copies are not made or distributed for profit or commercial advantage and that copies bear this notice and the full citation on the first page. To copy otherwise, or republish, to post on servers or to redistribute to lists, requires prior specific permission and/or a fee.

Copyright is held by the author/owner(s).

HT'04, August 9-13, 2004, Santa Cruz, California, USA

ACM 1-58113-848-2/04/0008.
The work described here is an experiment to investigate whether existing SCORM courseware can be augmented to provide personalised adaptive links for learners. The proposed solution involves the use of AuldLinky [4], a contextual open hypermedia FOHM server that provides dynamic links at runtime spanning over one or more FOHM linkbases. FOHM, the Fundamental Open Hypermedia Model, is a means of representing hypermedia information in a structured way and has a defined notion of context, and how that context affects views of the structure [5].

\section{SYSTEM ARCHITECTURE}

The architecture has several phases: the automatic generation of a concept map as a FOHM linkbase, the creation of an additional 'local linkbase' using specifically designed authoring tools, and the generation of adaptive links with AuldLinky. This is work in progress although the first phase of the architecture has already been implemented and there is considerable existing research that demonstrates the use of AuldLinky to supply adaptive links [5].

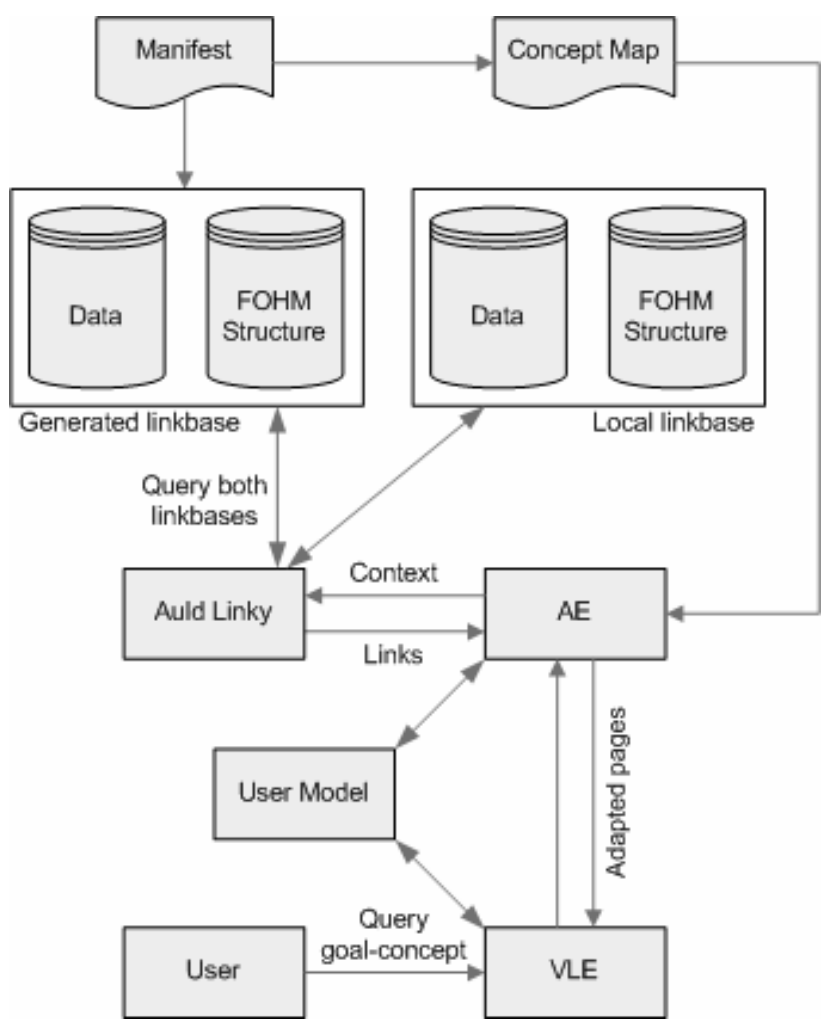

Figure 1. A proposed architecture to augment an existing SCORM manifest with personalized adaptive links using FOHM and AuldLinky. 


\subsection{Generating the Concept Map}

An XML concept map is used to represent the relationships between concepts in the manifest file. The map is used to specify the propagation of knowledge while learning. We assign each concept a name (derived from the title of the item node in the manifest) and an associated node id. We deduce the parent node id and any prerequisite information required to study this concept from the information in the manifest.

We make the assumption that each node in the manifest represents a node in the concept map, and that the title of each node is semantically meaningful so that it will be possible to create alternative links as described in 2.3 .

A weight is also assigned to each node to indicate the percentage contribution the concept at this node makes to its parent node. We must make some assumptions in order to assign this weight. In the simplest case of a hierarchical manifest we make the default assumption that each child node contributes equally to the understanding of the parent concept. However IMS Simple Sequencing introduces "rollup" rules at certain points which specify how the results of completing the current node (and it's children) should be propagated back up to parent nodes. The rule can contain statements such as "any", "all", "at least Y" of my children are needed to complete this node.

Unfortunately in current practice [6], the rollup rule elements are usually applied by assessments (objective tests) and the mapping of which questions demonstrate understanding of which child concepts is not explicit, so we must assume that all child nodes carry concepts that have an equal importance.

\subsection{Transformation to FOHM Objects}

Upon the completion of a concept map, the manifest is again traversed and the relevant elements are transformed into XMLbased FOHM objects. These FOHM objects model the structure of the manifest file as a set of typed FOHM associations (tours, concepts and level's of detail). For a description of these associations, the reader is referred to [5].

\subsection{Creating the Local Linkbase}

To provide added value to the existing PIF content, the new FOHM objects will be merged with locally-created linkbases. Our plan is to produce a simple authoring tool which will allow the author to browse the concept map and to identify links to additional materials which can be used as alternative presentations for given concepts (e.g. in order to satisfy differing learning styles) or to provide more detailed or supplementary information. The tool should provide functions that allow authors to include either their own materials or download external resources into their linkbases. However some kind of resource classifications and navigational control needs to be considered to correctly model them in FOHM.

A possible line of further work is to produce agents which attempt to automate the identification of suitable alternative resources in negotiation with learning object repositories.

\subsection{Adaptive Linking with AuldLinky}

With a version of the SCORM manifest converted into FOHM and locally-created linkbases, these structures can then be merged and queried in dynamic and adaptive ways. Previous work with the $\mathrm{HA}^{3} \mathrm{~L}$ system [5] has demonstrated how different forms of adaptive hypermedia techniques can be achieved through AuldLinky [4]. AuldLinky provides a storage and query mechanism for FOHM objects. By exploiting the complexity found within the FOHM model through the construction of a variety of specialised FOHM objects (such as tours, links, and levels of detail), an educational or adaptive runtime environment interacting with AuldLinky can provide many adaptive techniques such as adaptive link hiding, canned text adaptation and adaptive link annotation. This adaptation is achieved through the construction of specialized queries to AuldLinky that match context objects which can be attached to various points within the FOHM model. The resulting linkbase will then be processed by an adaptive engine that streams adapted pages to a browser, LMS or VLE (Virtual Learning Environment).

\section{CONCLUSION}

Our research proposes a method of dynamically adding adaptive links to a SCORM manifest to compliment its pre-existing materials. In this paper we have briefly described how a concept map and FOHM linkbases can be automatically generated from the manifest structure. Our future work will focus on developing the local linkbase authoring tool and merging these links with the existing educational material via an adaptive engine.

\section{REFERENCES}

[1] ADL SCORM. SCORM Version 1.3. Application Profile Working Draft 0.9, 27 November 2002, at http://xml.coverpages.org/SCORMV1.3-SeqAppProfile.pdf, last accessed 20 May 2004

[2] IMS. IMS Content Packaging Information Model Version 1.1.3 Final Specification, 2003, at http://www.imsglobal.org/content/packaging/, last accessed 20 May 2004

[3] Abdullah, N.A, \& Davis, H. Is Simple Sequencing Simple Adaptive Hypermedia?. In Proceedings of the $14^{\text {th }} \mathrm{ACM}$ Conference on Hypertext and Hypermedia, August 26-30, 2003, Nottingham, UK, pages pp. 172-173

[4] Michaelides, D.T., Millard, D.E., Weal, M.J. \& De Roure, D., C. Auld Leaky: A Contextual Open Hypermedia Link Server. In Proceedings of the 7th Workshop on Open Hypermedia Systems, ACM Hypertext 2001 Conference. Aarhus, Denmark 2001

[5] Bailey, C., Hall, W., Millard, D. E. \& Weal, M. J. Towards Open Adaptive Hypermedia. In Proceedings of 2nd International Conference, Adaptive Hypermedia and Adaptive Web-Based Systems 2347, pages pp. 36-46, Málaga, Spain. De Bra, P., Brusilovsky, P. and Conejo, R., Eds. 2002

[6] ADL Technical Team. SCORM Photoshop Examples Version 1.0, $31 \mathrm{Mac}$ 2004, http://www.adlnet.org 